\title{
Observation of second-harmonic generation in an oriented glassy nematic phase of a closo-decaborane derivative
}

\author{
Andrzej Miniewicz, ${ }^{\text {a) }}$ Anna Samoc, and Marek Samoc \\ Laser Physics Centre, Research School of Physical Sciences and Engineering, The Australian National \\ University, Canberra, Australian Capital Territory 0200, Australia \\ Piotr Kaszynski \\ Organic Materials Research Group, Chemistry Department, Vanderbilt University, \\ Nashville, Tennessee 37235
}

(Received 6 December 2006; accepted 27 June 2007; published online 14 August 2007)

\begin{abstract}
The optical second-harmonic generation (SHG) and the linear optical properties were investigated in a planar cell containing aligned nonchiral nematic derivative of $\mathrm{B}_{10} \mathrm{H}_{10}^{2-}$ : 1-(4-heptyloxypyridin-1-yl)-10-(4-pentyl-1-thiacyclohexyl)-closo-decaborane (HPTD) as a function of temperature. The compound showed a low level of the SHG signal in the polycrystalline state, and no nonlinear optical effect was detected in the fluid nematic state $(N)$ either in the presence or absence of an orienting electric field. When frozen, the resulting thermodynamically unstable nematic glassy phase (glassy $N$ ) unexpectedly exhibited the second-order nonlinear optical properties, implying a partial polar order in the arrangement of the in-plane-aligned molecules in a liquid-crystal cell. The efficiency of the SHG was analyzed for two polarization configurations yielding the main component of the second-order nonlinear susceptibility $\chi_{x x x}^{(2)}=2.3 \pm 0.3 \mathrm{pm} \mathrm{V}^{-1}$ along the nematic director $x$. A comparison of the experimental susceptibility with a hypothetical one estimated from the vectorial component of the molecular hyperpolarizability showed a relatively low degree of the spontaneous polar order. The dispersion of the principal refractive indices in the glassy state of HPTD was derived from dichroic absorption spectra using an interference fringe method and fitted with a Sellmeier-type equation. At $527 \mathrm{~nm}$ the birefringence of HPTD is $\Delta n=0.31$. ( 2007 American Institute of Physics. [DOI: 10.1063/1.2767868]
\end{abstract}

\section{INTRODUCTION}

Optical properties of liquid crystals (LCs) continue to be of fundamental interest and to play a prominent role in contemporary technologies. ${ }^{1-3}$ Of particular interest are the nonlinear optical (NLO) properties of LCs, and both secondorder and third-order nonlinear effects have been observed in these organized liquids. ${ }^{4-6}$

Generation of the second harmonic (SHG) of the frequency of light is one of the simplest manifestations of second-order optical nonlinearity. ${ }^{7}$ In the SHG process, the NLO material converts a light wave of frequency $\omega$ into a light wave of frequency $2 \omega$. The relevant second-order optical property is described by a second-order susceptibility tensor $\chi_{i j k}^{(2)}(-2 \omega ; \omega, \omega)$ that has nonvanishing components only for macroscopically noncentrosymmetric materials. The nematic liquid crystals that possess axial $\left(D_{\infty}\right)$ ordering are centrosymmetric and do not exhibit the bulk second-order NLO properties. However, some smectic phases, such as the ferroelectric $\mathrm{SmC}^{*}$ phase, are capable of harmonic light conversion, although usually with a small efficiency. ${ }^{8-12}$

In this paper, we describe the spontaneous formation of NLO active ordered glass from a nematic phase of nonchiral 1-(4-heptyloxypyridin-1-yl)-10-(4-pentyl-1-thiacyclohexyl)-

\footnotetext{
a) Author to whom correspondence should be addressed; present address: Institute of Physical and Theoretical Chemistry, Wroclaw University of Technology, 50-370 Wroclaw, Poland; FAX: +48-71-3203364; electronic mail: andrzej.miniewicz@pwr.wroc.pl
}

closo-decaborane (HPTD) (Fig. 1). We have probed the polar order in this material by investigating the SHG signal induced with 7 ps laser pulses at $\lambda=1053 \mathrm{~nm}$.

The nematogenic HPTD (Fig. 1) is an unusual compound. It is derived from the $\mathrm{B}_{10} \mathrm{H}_{10}^{2-}$ inorganic cluster and belongs to a class of liquid crystals containing closo-borane cages in the rigid core. ${ }^{13,14}$ HPTD has been designed specifically to exhibit liquid crystalline properties and directional intramolecular electron transfer (cage to pyridine) upon photoexcitation. ${ }^{15}$ This symmetry-allowed electronic transition along the long molecular axis $(\hat{\xi}=3)$ shows up as the lowest energy band in the ultraviolet (UV) spectrum with a peak at $301 \mathrm{~nm}$ in acetonitrile. ${ }^{14}$ The dissymmetric substitution of $\mathrm{B}_{10} \mathrm{H}_{10}^{2-}$ with two onium groups in HPTD results in a

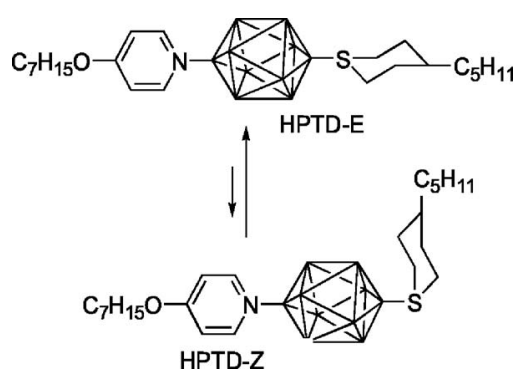

FIG. 1. The trans isomer (HPTD-E) and cis isomer (HPTD-Z) of 1(4-heptyloxypirydin-1-yl)-10-(4-pentylthiacyclohex-1-yl)-closo-decaborane. Each equatorial vertex of the cage represents a $\mathrm{BH}$ (boron-hydrogen) fragment, and the substituents are connected directly to the apical boron atoms. 
modest longitudinal net dipole moment calculated to be 5.0 D, which consequently gave rise to a modest positive dielectric anisotropy of the material (more details will be provided in a separate publication). Crystallographic studies of HPTD at $-100{ }^{\circ} \mathrm{C}$ showed a centrosymmetric crystal structure. The differential scanning calorimetry (DSC) measurements show that HPTD melts at $115^{\circ} \mathrm{C}$ to form a nematic $(N)$ phase, which subsequently becomes an isotropic $(I)$ liquid at $158{ }^{\circ} \mathrm{C}$. On cooling, a large hysteresis is observed and the nematic fluid phase glassifies significantly below the melting point. The nematic glass (glassy $N$ ) crystallizes slowly and the rate of crystallization depends on the rate of cooling. With fast cooling rates $\left(>20{ }^{\circ} \mathrm{C} / \mathrm{min}\right)$ the glass phase exists for up to $2 \mathrm{~h}$ at room temperature before visible crystallites begin to form. The inhibition of crystallization and the ease of formation of this glassy state by HPTD have been attributed to the high viscosity of the material and the presence of a high percentage of the bent cis isomer HPTD$Z$.

We report here the second-order nonlinear optical effects in the supercooled glassy nematic phase of HPTD confined in a microstructured thin cell with the imposed planar alignment. We measure the intensity and the anisotropy of the second-harmonic light spontaneously generated in this phase in a broad temperature range.

\section{EXPERIMENTAL DETAILS AND PROCEDURES}

The preparation of HPTD and the full thermal, dielectric, and spectroscopic properties as well as the crystal and molecular structures will be described elsewhere. The optical properties of HPTD were measured in a $9.1 \mu \mathrm{m}$ glass cell obtained from LC Vision ${ }^{\circledR}$. The cell had indium tin oxide (ITO) conductive layers, which were covered with obliquely evaporated $\mathrm{SiO}_{2}$ layers to impose the planar molecular alignment along the $x$ direction. The cell was filled with HPTD by capillary action at $\sim 130{ }^{\circ} \mathrm{C}$. The coordinate system $x y z$ is that of the LC cell. The $z$ axis was chosen to be perpendicular to the plane of the glass plates.

The absorption spectrum of polycrystalline HPTD in the cell was measured in the wavelength range of 200-3200 nm with a Shimadzu model UV-3100PC spectrophotometer at room temperature $\left(21-22{ }^{\circ} \mathrm{C}\right)$. Results showed that HPTD is transparent in the entire visible and near-infrared (NIR) part of the spectrum. This includes the region between 1300 and $1600 \mathrm{~nm}$, which is important in telecommunication, and $500-550 \mathrm{~nm}$ in which the second harmonic $(526.5 \mathrm{~nm})$ of the fundamental wave of the laser used in these studies (1053 nm) appears.

The refractive index of HPTD in its glassy state was determined at $22{ }^{\circ} \mathrm{C}$ using a Varian Cary model 5000 ultraviolet-visible/near-infrared scanning spectrophotometer and a method similar to that of Swanepoel. ${ }^{16-18}$ The glassy nematic sample was prepared by heating the cell containing HPTD to about $120^{\circ} \mathrm{C}$, followed by rapid cooling to the ambient temperature at which the measurements were performed within an hour. The measurement method exploits a relationship between the dispersion of the refractive index $n(\lambda)$ and the positions of interference fringes, which were

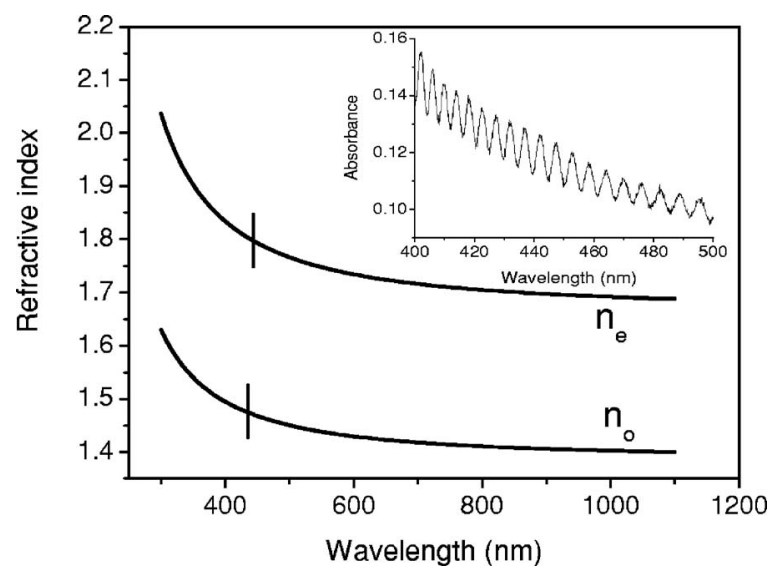

FIG. 2. The dispersion of the refractive indices $n_{e}(\lambda)$ and $n_{o}(\lambda)$ measured in the ordered glassy nematic state of HPTD by the interference fringe method. Uncertainty of the index measurements equal to \pm 0.05 is represented by the vertical bars in the graph. The inset shows the interference fringes observed in the absorbance spectra being measured with the light polarized parallel to the groove alignment direction.

observed during the absorption $A(\lambda)$ measurements of a thin film of HPTD in the liquid-crystal cell in a wide spectral range. The interference fringes were recorded for two polarizations of the incoming light: along the alignment direction $x$ of the grooves in the cell (the $\mathbf{E}$ vector of the light beam was polarized parallel to the groove direction) and along the $y$ direction (the $\mathbf{E}$ vector of the light beam was polarized perpendicular to the groove alignment direction), which corresponded to measurements of the extraordinary $n_{e}(\lambda)$ and the ordinary $n_{o}(\lambda)$ refractive indices of the sample, respectively (see inset of Fig. 2). Each set of $A(\lambda)$ fringes was fitted in the entire spectral range using the Sellmeier twoparameter refractive index dispersion equation in which $A$ and $\lambda_{0}$ are constants,

$$
n(\lambda)^{2}=1+\left(\frac{A \lambda^{2}}{\lambda^{2}-\lambda_{0}^{2}}\right) .
$$

With the known thickness of the empty cell ( $d$ $=9.1 \mu \mathrm{m})$, the analysis of the spectra was performed with several dozens of the fringes in the range of 380-900 nm. The parameters $A$ and $\lambda_{0}$ for the refractive index dispersion relations were obtained with the procedure of fitting of the wavelength positions of the absorbance fringes with the equation:

$$
A(\lambda)=\sin \left[\frac{2 \pi n(\lambda)}{\lambda} d+\Delta \varphi_{0}\right]
$$

by applying a relative phase $\Delta \varphi_{0}$ and matching them to the wavelength positions of the measured fringes. This has led to the dispersion relations (3a) for the extraordinary index $n_{e}$ and $(3 \mathrm{~b})$ for the ordinary $n_{o}$ refractive index of HPTD at room temperature,

$$
n_{e}(\lambda)^{2}=1+\left[\frac{1.79 \lambda^{2}}{\lambda^{2}-(197)^{2}}\right]
$$




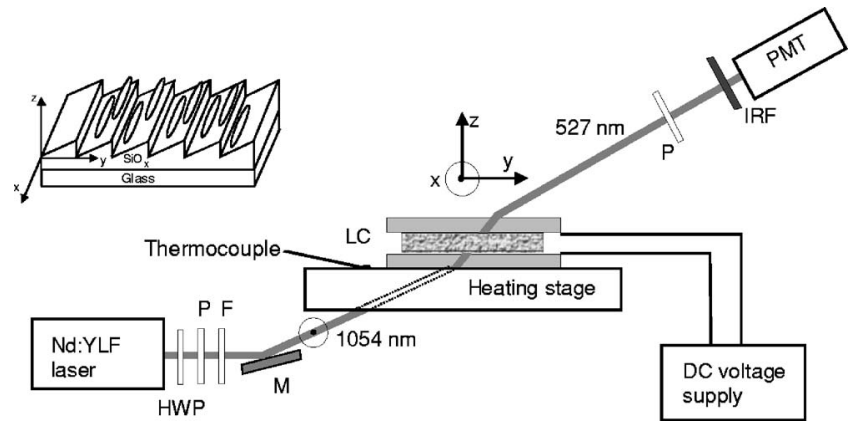

FIG. 3. A diagram of the experimental setup for the SHG measurements (a side view). The $1053 \mathrm{~nm}$ laser beam enters the stage from below at approximately $40^{\circ}$. The light beam has $s$ polarization described by $\hat{\mathbf{E}}$ vector, as shown by a circle. Inset shows a schematic of molecular ordering on $\mathrm{SiO}_{2}$ substrate. PMT, photomultiplier tube; P, polarizer; F, filter; IRF, infrared cut-off filter; HWP, half-wave retardation plate; and LC, liquid-crystal cell filled with HPTD. The temperature was measured with a thermocouple glued to the stage close to the sample.

$$
n_{o}(\lambda)^{2}=1+\left[\frac{0.929 \lambda^{2}}{\lambda^{2}-(199)^{2}}\right]
$$

where $A_{e}=1.79, \lambda_{0, e}=197 \mathrm{~nm}$ and $A_{o}=0.929, \lambda_{0, o}=199 \mathrm{~nm}$ have been found for $n_{e}$ and $n_{o}$, respectively. The dispersion curves (3a) and (3b) are shown in Fig. 2.

The birefringence of HPTD, $\Delta n(\lambda)=n_{e}(\lambda)-n_{o}(\lambda)$, was calculated using relations (3a) and (3b) at the relevant wavelengths: $\Delta n=0.31$ at $526.5 \mathrm{~nm}$ and $\Delta n=0.29$ at $1053 \mathrm{~nm}$. This is a substantially large birefringence. It is consistent with the theoretical $\Delta n=0.29$ obtained from the calculated electronic polarizabilities [by modified neglect diatomic overlap (MNDO) method] for the pure trans isomer (HPTD$E)$ and the Vuks model, ${ }^{19}$ assuming order parameter $S=0.8$ and density $\rho=1.05 \mathrm{~g} / \mathrm{cm}^{3}$.

The qualitative SHG measurements as a function of temperature were performed for three identical LC cells containing HPTD using a setup shown in Fig. $3 .^{20-22}$

The SHG measurements were performed in the experimental geometries G1 and G2. In the G1 geometry the LC cell filled with HPTD material was placed on a temperaturecontrolled stage. The pulse laser beam of the fundamental wavelength of $1053 \mathrm{~nm}$ entered the sample from below at an angle of approximately $40^{\circ}$. This beam of fundamental frequency $\omega$ was delivered by a semiconductor saturable absorber mirror (SESAM) mode-locked diode-pumped neodymium-doped yttrium lithium fluoride (Nd:YLF) laser followed by a regenerative $\mathrm{Nd}$ :YLF amplifier operating at $1053 \mathrm{~nm}$, with the repetition rate of $20 \mathrm{~Hz}$, pulse width of about 7 ps, and energy of about $100 \mu \mathrm{J}$. The pulse width was determined in a separate experiment by observation of selfdiffraction autocorrelation in a poly( $p$-phenylenevinylene) (PPV) thin film. The incident fundamental beam was linearly polarized with the $\hat{\mathbf{E}}$ vector of light oscillating parallel to the sample substrate ( $s$ polarization, $\hat{\mathbf{E}} \| x$ ). The beam was unfocused and no collecting lens was used for the detection of the second-harmonic light $(2 \omega)$. A long-pass filter was placed immediately before the sample to block any spurious secondharmonic light generated by optical components of the laser system. A photomultiplier (PMT) equipped with filters blocking the beam of the fundamental wavelength and leaving only the second-harmonic light generated by the sample at $526.5 \mathrm{~nm}$ (a green color filter followed by a narrow band interference filter) was used as a detector. In some experiments a static electric field perpendicular to the sample surfaces (i.e., in the $z$ direction) was applied by means of the ITO electrodes. Its maximum strength was $\left|E_{\mathrm{dc}}\right|= \pm 3.3$ $\times 10^{6} \mathrm{~V} / \mathrm{m}( \pm 30 \mathrm{~V}$ applied to the cell $)$.

The polarization dependence of the SHG signals was measured in the experimental geometry G2, where the sample was placed on a rotary stage with the $x y$ plane set perpendicularly to the incident fundamental frequency beam (the $z$ axis was the sample rotation axis). The laser beam was vertically polarized $(\hat{\mathbf{E}} \| x)$. A sheet polarizer was placed before the entrance slit of the photomultiplier to read the $x$ and $y$ polarized components of the SHG signal emerging from the sample during its rotation by $360^{\circ}$ with a $5^{\circ}$ step. The strongest SHG signal was always observed for the fundamental light polarized parallel to the groove direction (the $x$ axis) in the planar LC cell, which corresponded to the preferred molecular alignment of the liquid-crystal director $\hat{n}$ in the nematic phase. The photomultiplier current output data were collected and averaged over several tens of laser shots.

The SHG in HPTD (G1 geometry) was studied in situ as a function of temperature during heating and cooling. In all experiments involving a fast temperature change, the temperature recorded by the thermocouple was generally a poor approximation of the temperature of the HPTD sample, being either lower during heating or higher during cooling than the actual temperature of the sample.

Quantum-mechanical calculations were carried out using the MNDO algorithm ${ }^{23}$ implemented in the MOPAC 93 computational package. The geometry of the HPTD- $E$ isomer was fully optimized in the most extended conformation using the PRECISE keyword.

\section{RESULTS}

The generation of the second-harmonic signal in a sample of HPTD in the LC cell was investigated both with and without the applied external dc electric field in the geometry G1. Then the anisotropy of the SHG signal was investigated only in the absence of the dc electric field in the geometry G2.

The first set of the SHG experiments was performed with HPTD in a LC cell without the external dc electric field in the geometry G1 (see Fig. 4). Measurement of an opaque polycrystalline sample at room temperature gave a weak but detectable SHG signal.

Upon heating, the SHG signal abruptly disappeared close to the $\mathrm{Cr}-\mathrm{N}$ transition $\left(\sim 115^{\circ} \mathrm{C}\right)$ and, as expected, the NLO effect was not observed in the nematic phase. Surprisingly, however, upon cooling of the nematic phase below the melting point $\left(\sim 115^{\circ} \mathrm{C}\right)$, a weak SHG signal reappeared and grew stronger with the decreasing temperature reaching much higher second-harmonic intensity values than those previously observed at room temperature. The appearance of the SHG signal coincided with the formation of a translucent glassy state ascribed to a "nematic glass" because only a 


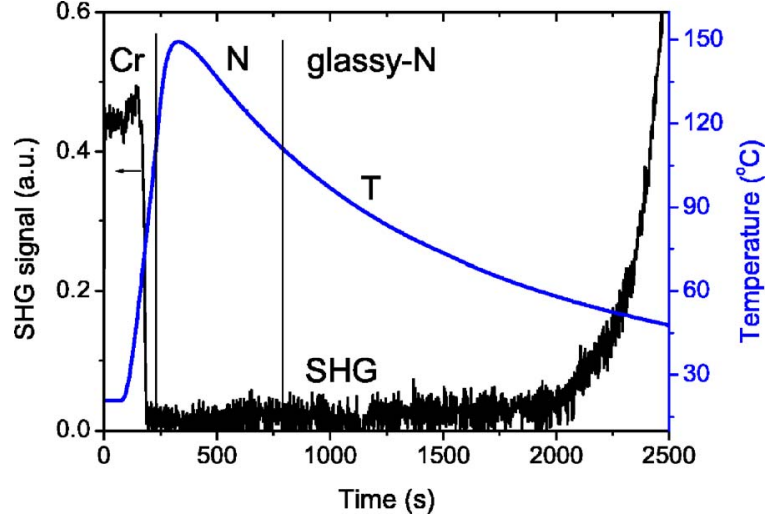

FIG. 4. The SHG signal for HPTD as a function of time during the first fast heating and cooling cycle. The heating rate was approximately $40{ }^{\circ} \mathrm{C} / \mathrm{min}$ and averaged cooling rate was $3.3^{\circ} \mathrm{C} / \mathrm{min}$. The fundamental beam was $s$ polarized and no external electric field was applied during this experiment.

slight change was observed in the schlieren optical texture. The subsequent heating of the sample showed a reverse process: the SHG signal was decreasing and disappeared completely when the temperature approached $95^{\circ} \mathrm{C}$. This result was well reproducible in the multiple heating or cooling cycles between the glassy and the nematic phases of the material heated with the rate of about $20^{\circ} \mathrm{C} / \mathrm{min}$ and cooled with about $4{ }^{\circ} \mathrm{C} / \mathrm{min}$ (Fig. 5). Practically no secondharmonic intensity hysteresis was observed in the repeated heating or cooling cycles. The onset of the SHG signal was close to $95{ }^{\circ} \mathrm{C}$ (i.e., $20{ }^{\circ} \mathrm{C}$ below the $\mathrm{Cr}-\mathrm{N}$ transition), and the intensity of the signal practically leveled off at about $70{ }^{\circ} \mathrm{C}$. Further cooling of the sample did not result in any significant increase of the signal. After leaving the sample at room temperature overnight the intensity of the SHG signal decreased, possibly due to the increase of light scattering caused by the crystallization seen even by a naked eye. Nevertheless, the polycrystalline sample still showed a residual SHG signal after storing for several days at ambient temperature.

In the second set of the SHG experiments (G1 geometry), the dc electric field was applied to the sample through ITO electrodes, i.e., in the cell $z$ direction, and the second-

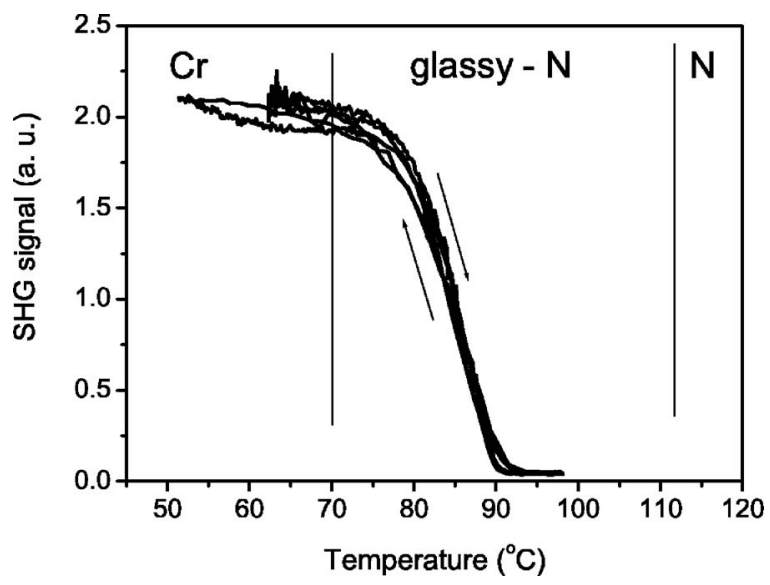

FIG. 5. SHG signal recorded for HPTD under the same conditions as in Fig. 4 for several heating/cooling cycles. The average heating rate was $20{ }^{\circ} \mathrm{C} / \mathrm{min}$ and the cooling rate was $4{ }^{\circ} \mathrm{C} / \mathrm{min}$.

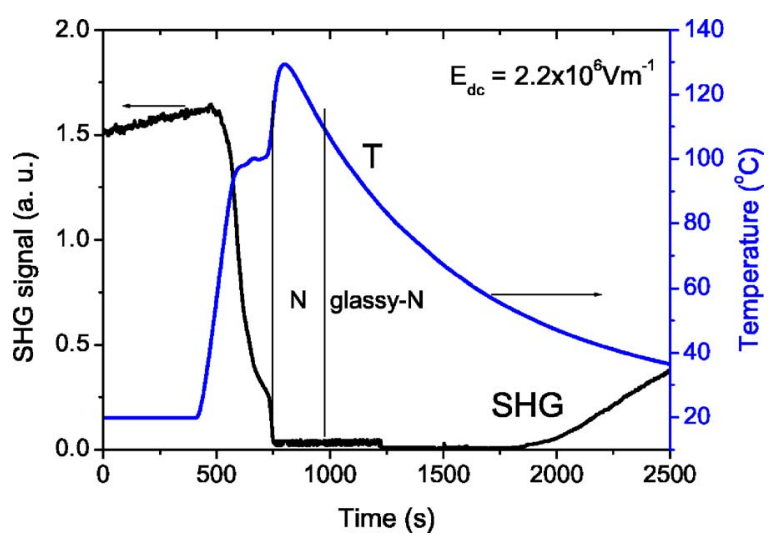

FIG. 6. The SHG signal was measured with $p$-polarized fundamental beam as a function of temperature in HPTD in a $9.1 \mu \mathrm{m}$ thick cell with applied external electric field of $2.2 \times 10^{6} \mathrm{~V} \mathrm{~m}^{-1}$ (i.e., $+20 \mathrm{~V}$ applied across the layer thickness).

harmonic intensity was measured with the fundamental light $s$ polarized. Previously, no SHG was detected in the nematic phase even in the presence of electric field $E_{\mathrm{dc}}=2.2$ $\times 10^{6} \mathrm{~V} \mathrm{~m}^{-1}$ (Fig. 6). Upon cooling to the glassy phase, the second harmonic appeared but its intensity was only about $17 \%$ of that for the unbiased sample under the same experimental conditions. This result can be rationalized by a homeotropic orientation of molecules in the nematic phase induced by the applied electric field of $2.2 \times 10^{6} \mathrm{~V} \mathrm{~m}^{-1}$ $(20 \mathrm{~V} / 9.1 \mu \mathrm{m}$ LC layer) that significantly exceeded the threshold voltage $V_{\mathrm{TH}}$ of the sample $(5.6 \mathrm{~V})$. The homeotropic nematic formed the glassy phase with molecules preferentially aligned in the $z$ direction, which diminished their effective interaction with the incident $s$-polarized laser beam.

Since a static electric field was found to affect the intensity of the SHG signal in the glassy HPTD, it was interesting to test whether the SHG signal could be modulated by the dc electric field applied in the temperature range in which the SHG signal dynamically changes its value $\left(70-90^{\circ} \mathrm{C}\right)$. Indeed, application of the voltage of $30 \mathrm{~V}$ to the sample near the glass-nematic transition resulted in a $10 \%$ decrease of the SHG signal intensity, while removing the voltage restored the SHG intensity. The switching time was about $2-3 \mathrm{~s}$ (Fig. 7). The influence of the electric field on the observed signal became progressively less detectable, and the overall intensity of the signal decreased with increasing number of cycles. This can be attributed to a relatively rapid crystallization in this temperature range. The same experiment repeated at $50{ }^{\circ} \mathrm{C}$ showed no effect of the electric field on the SHG signal, apparently due to the already rigid structure of glassy HPTD.

In order to quantify the SHG susceptibility of the measured sample, the signal was analyzed in the G2 geometry using one of the two configurations: (i) both fundamental and second-harmonic beams are polarized along the $x$ axis and (ii) fundamental beam is polarized along the $x$ axis and second harmonic along the $y$ axis. The sample was positioned with its face normal to the incident $1053 \mathrm{~nm}$ beam with $\overrightarrow{\mathbf{E}}$ vector of light along the groove rubbing direction $x$. The measured second harmonic was analyzed with an effective nonlinear susceptibility $\chi_{\text {eff }}^{\mathrm{LC}}$ relation, ${ }^{24}$ 


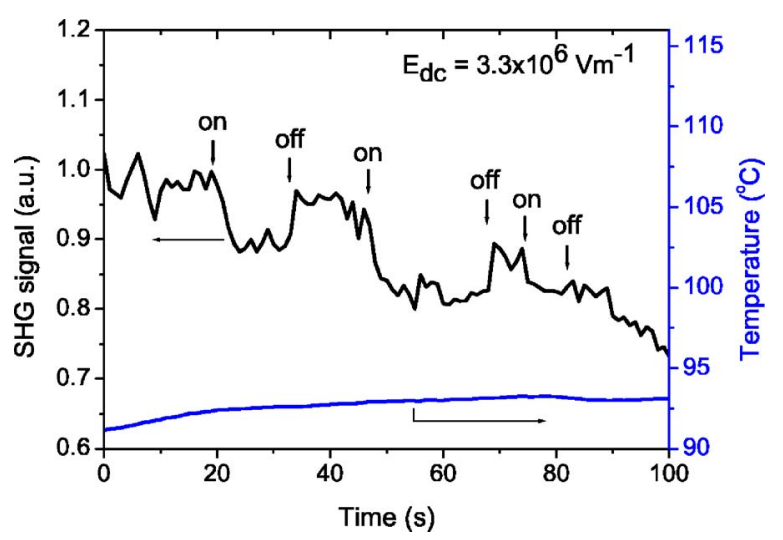

FIG. 7. The effect of electric field $\left(\left|E_{\mathrm{dc}}\right|=3.3 \times 10^{6} \mathrm{~V} \mathrm{~m}^{-1}\right)$ on the intensity of the spontaneously generated SHG signal in the glassy nematic phase of HPTD at about $92{ }^{\circ} \mathrm{C}$. The lower line monitors the temperature during the experiment. Experimental conditions were the same as in Fig. 4.

$$
\chi_{\mathrm{eff}}^{\mathrm{LC}}=\hat{\mathbf{e}}(2 \omega) \cdot \chi^{(2)}: \hat{\mathbf{e}}(\omega) \cdot \hat{\mathbf{e}}(\omega),
$$

where $\hat{\mathbf{e}}(\omega)$ and $\hat{\mathbf{e}}(2 \omega)$ are the unit polarization vectors of the output and the input light, respectively, and $\chi^{(2)}$ $(-2 \omega ; \omega, \omega)$ denotes the second-order susceptibility tensor. For the (i) light polarization configuration $\hat{\mathbf{e}}_{\text {in }}(\omega)=\hat{\mathbf{e}}_{\text {out }}(2 \omega)$ $=(1,0,0)$ and for the (ii) another one $\hat{\mathbf{e}}_{\text {in }}(\omega)=(1,0,0)$ but $\hat{\mathbf{e}}_{\text {out }}(2 \omega)=(0,1,0)$. The second-harmonic intensity obtained from the HPDT material in the LC cell in such condition was compared to the second-harmonic intensity at the strongest Maker fringe from the $2 \mathrm{~mm}$ thick Y-cut quartz plate [for which $d_{11}=(1 / 2) \chi_{111}^{(2)}=0.5 \mathrm{pm} \mathrm{V}^{-1}$ was assumed]. ${ }^{25}$ For equal incident fundamental beam intensities, $\chi_{\mathrm{eff}}^{\mathrm{HPTD}}$ of liquid crystal can be deduced from ${ }^{25,26}$

$$
\chi_{\mathrm{eff}}^{\mathrm{HPTD}}=\chi_{11}^{\text {quartz }}\left[\left(\frac{I_{\mathrm{SHG}}^{\mathrm{HPTD}} \cdot \eta^{\mathrm{HPTD}}}{I_{\mathrm{SHG}}^{\text {quartz }} \cdot \eta^{\text {quartz }}}\right)^{1 / 2}\right] \frac{L_{c}^{\text {quartz }}(0)}{L_{c}^{\mathrm{HPTD}}(0)},
$$

where $L_{c}^{\mathrm{HPTD}}(0)$ and $L_{c}^{\text {quartz }}(0)$ are the coherence lengths at normal incidence defined generally as $L_{c}(0)=\pi c / 2 \omega\left(n_{2 \omega}\right.$ $\left.-n_{\omega}\right)$, and $\eta^{\mathrm{HPTD}}=6.482$ and $\eta^{\text {quartz }}=4.184$ are the coefficients defined as $\eta=\left(n_{\omega}+1\right)^{3}\left(n_{2 \omega}+1\right)^{3}\left(n_{2 \omega}\right.$ $\left.+n_{\omega}\right) / 128 n_{2 \omega} R(0)$ with $R(0)$ being the multiple reflection factor at normal incidence. From the comparison of the experimental SHG signal efficiencies measured at the same voltage $(900 \mathrm{~V})$ applied to the PMT, we found $I_{\mathrm{SHG}}^{\mathrm{HPTD}} / I_{\mathrm{SHG}}^{\text {quartz }}$ $=1 / 8$. Then, from the formula (5), with assumed value of $R(0) \cong 1$ and calculated values of $L_{c}^{\mathrm{HPTD}} \approx 4 \mu \mathrm{m}$, and taking $L_{c}^{\text {quartz }}(0) \approx 20.65 \mu \mathrm{m}$, we obtained for the (i) polarization configuration $\chi_{\mathrm{eff}}^{\mathrm{HPTD}} \approx 2.3 \pm 0.3 \mathrm{pm} \mathrm{V}^{-1}$. This value represented only an estimate (in fact, a lower limit) of the susceptibility value because the sample was a nonphase-matchable SHG material and its effective thickness was not optimized (e.g., by varying the incidence angle, as it was typically done in Maker fringe measurements) in order to be an exact odd multiple of the coherence length.

The anisotropy of the glassy state of the mesogenic HPTD was probed with the sample being rotated perpendicular to the direction of the incident fundamental beam. The analyzer was set either for (i) or (ii) polarization configuration in the SHG experimental geometry G2, while the second-harmonic signal was measured. The resulting polar

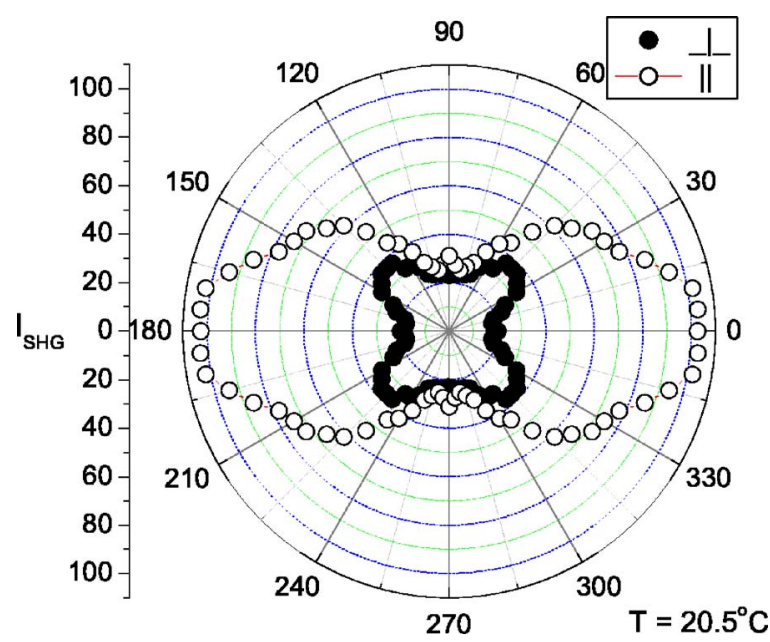

FIG. 8. The second-harmonic intensity was measured at the azimuthal angle $\Phi$ by which a sample of HPTD is rotated. The electric field of the incoming fundamental wave $\hat{\mathbf{E}}$ was parallel to the alignment direction for the $\Phi=0^{\circ}$. The outcoming SHG light was analyzed with a polarizer set perpendicular (black circles) or parallel (open circles) to the $\hat{\mathbf{E}}$ vector of the incoming linearly polarized fundamental light beam.

plot of the second-harmonic intensity is shown in Fig. 8. The azimuthal angle $\Phi$ describes the sample rotation, and the $\Phi$ $=0^{\circ}$ corresponds to the incident beam polarization being parallel to the alignment direction $x$. The second-harmonic intensity $I_{\mathrm{SHG}}$ shows a pronounced anisotropic dependence on the sample orientation; the intensity of the SHG signal was about four times larger in the direction parallel to the nematic director $x$ than in the perpendicular direction $y$. An in-plane unidirectional alignment (such as that imposed by the $\mathrm{SiO}_{2}$ layer on nematic liquid crystals) along the $x$ axis imposes the $\left(\infty \mathrm{mm}\right.$ or $\left.C_{\infty v}\right)$ symmetry of the system, which impacts the form of the $\chi^{(2)}$ tensor.

In general, there are five nonvanishing susceptibility elements, of which three are unique: $\chi_{x x x}^{(2)}, \chi_{x y y}^{(2)}=\chi_{x z z}^{(2)}$, and $\chi_{y y x}^{(2)}$ $=\chi_{z z x}^{(2)}$. For the (i) configuration the $\chi_{x x x}^{(2)}$ is readily accessible,

$$
\chi_{\mathrm{eff}}^{(2),(i)}=\chi_{x x x}^{(2)},
$$

while $\chi_{x y y}^{(2)}$ can be estimated in (ii) configuration after sample rotation by angle $\Phi=90^{\circ}$. The level of the isotropically scattered light is given by the depth of minimum of the secondharmonic signal measured in the (i) configuration for rotation by an angle $\Phi=90^{\circ}$ and amounts to $20 \%$ of the maximum SHG signal.

In order to gain insight in the possible role of the surface alignment on the formation of the polar glassy phase and the anisotropy of the NLO properties of HPTD in the LC cell, the material has also been investigated on untreated glass substrates (i.e., between fresh microscope cover glasses). A weak SHG signal appeared in both glassy and polycrystalline phases; however, the anisotropy of the signal could not be detected.

\section{DISCUSSION}

The appearance of the SHG signal in the glassy nematic phase of HPTD is surprising. The nematic mesophase is centrosymmetric and the nematic glass formed by a rapid tem- 
perature decrease is also supposed to be centrosymmetric and, therefore, not contributing to the SHG signal. Seeking possible reasons of the observed NLO activity in the rigid phase of HPTD one can consider two possibilities: (i) formation of noncentrosymmetric nanometer-size crystallites composed of HPTD- $E$ molecules-ordered within the nematic glassy phase or (ii) formation of a noncentrosymmetric structure composed of HPTD- $E$ with important contribution of bent HPTD- $Z$ molecules. If the presence of crystallites is assumed their size would need to be less than a fraction of the wavelength because no excessive light scattering is observed in the SHG active phase. Support for this concept might be offered by the results shown in Fig. 7. The observed reduction of the SHG signal from the glassy nematic phase of HPTD at $92{ }^{\circ} \mathrm{C}$ in the presence of the electric field is consistent with the assumption that the small size crystallites responsible for the NLO effect are able to reorient together with the matrix, which is partially fluid at this temperature. On removal of the electric field the previous planar alignment is partially recovered, as long as the viscosity of the glassy phase allows for it. For the optical field polarized along the director of a planarly aligned nematic, the electric field applied through the cell electrodes reorients the material and reduces the effective coupling of the optical field with the crystallites. Clearly, the crystallites with their optical axis lying perpendicular to the optical electric field would give a much smaller SHG response. Estimates of the transverse component of the molecular hyperpolarizability tensor of HPTD performed by MNDO indicate that it is approximately one order of magnitude smaller than the longitudinal component.

Let us estimate the maximum value of the second-order susceptibility component $\chi_{x x x}^{(2)}$ expected for a solid with totally polar oriented molecules of HPTD-E. The macroscopic second-order susceptibility $\chi_{i j k}^{(2)}(-2 \omega ; \omega, \omega)$ can be expressed in terms of the molecular hyperpolarizability ${ }^{27}$ and, therefore, can be estimated from the known $\beta_{I J K}(-2 \omega ; \omega, \omega)$ and the structure of the material. A hypothetical maximum value of the second-order susceptibility tensor component would represent a situation in which all NLO molecules are strictly aligned along the preferred polar orientation (here, the $x$ axis) with their dipole moments being parallel. Then, within the oriented gas approximation, the following relation holds: $\chi_{x x x}^{(2)} \propto N f \beta_{\xi \xi \xi}$, where $f$ represents the product of local field factors. With the value of molecular hyperpolarizability $\beta_{\xi \xi \xi}^{\mathrm{HRS}}=45 \pm 10 \times 10^{-30}$ esu $\left(1050 \times 10^{-40} \mathrm{~m}^{4} \mathrm{~V}^{-1}\right)$ measured for HPTD- $E$ by hyper-Rayleigh scattering (HRS) method at $1064 \mathrm{~nm}$ (to be published elsewhere), and taking the composite local field factor $f=4.439$ and number density $N$ $=1.36 \times 10^{27} \mathrm{~m}^{-3}$ from crystallographic data, the hypothetical susceptibility $\chi_{x x x}^{(2)}$ would be estimated as $\left|\chi_{x x x}^{(2)}\right|$ theor $\approx 640 \mathrm{pm} / \mathrm{V}$. Conversely, for an ideal antiparallel dipolar arrangement the same formula gives $\left|\chi_{x x x}^{(2)}\right|_{\text {theor }}^{\text {HRS }}=0$. The ratio between the maximal theoretically possible susceptibility value $\left|\chi_{x x x}^{(2)}\right|$ theor and the experimentally observed $\chi_{x x x}^{(2)}$ $=2.3 \mathrm{pm} / \mathrm{V}$ is nearly $300: 1$, which indicates that only a fraction of the molecular optical nonlinearity is effectively exploited.

The second possibility mentioned above (ii) was that of the formation of a noncentrosymmetric structure composed of HPTD- $E$ with important contribution of bent HPTD- $Z$ molecules. This is difficult to prove at this stage, as no clear independent indication of existence of a noncentrosymmetric HPTD structure has emerged. The possible role of the cis isomer HPTD- $Z$ in the generation of the SHG signal is not clear. All molecules in the solid-state structure of HPTD are the trans isomers, but in a liquid phase a trans-cis equilibrium quickly establishes due to a low activation energy for epimerization at the $S$ center $\left(\Delta G_{298}^{+t}=99.4 \pm 2 \mathrm{~kJ} / \mathrm{mol}\right)$. Thermodynamic studies of HPTD in toluene solutions indicate that the ratio of trans to cis isomers is about 7:3 in the nematic phase, and the isomers interconvert with a rate of $k$ $\approx 10 \mathrm{~s}^{-1}$ at $115^{\circ} \mathrm{C}$. Crystallization of the sample either from melt or from solution must proceed with a configurational selection for the trans isomer HPTD- $E$. A large concentration of the cis isomer HPTD- $Z$ within the nematic phase of HPTD apparently inhibits the crystallization process, and a nematic glass is readily formed from the melt upon cooling. It is possible that the crystallization taking place under these conditions in a very viscous phase may result in the HPTD- $Z$ molecules being incorporated into the crystal lattice. The crystal structure of HPTD obtained at $-100{ }^{\circ} \mathrm{C}$ is centrosymmetric, which cannot exhibit any second-order nonlinear optical effects. All molecules in the solid-state structure of HPTD are trans isomers. It is possible, however, that there are other polymorphs of HPTD that either coexist with that of the analyzed single crystal or exist as high-temperature crystalline noncentrosymmetric modifications. Therefore one can speculate that these other crystalline polymorphs, which can be formed with the help of the cis isomer, would be responsible for the appearance of the weak SHG signal observed both in the nematic glass and in the polycrystalline solid. For the polycrystalline solid the SHG signal intensity is about ten times smaller than for the glassy nematic phase. It is possible that both the crystalline solid and the glass have similar levels of polar order, and the extensive light scattering by the polycrystalline structure is responsible for the observed SHG intensity difference.

An effort to resolve which of the two hypotheses is more probable is undertaken by investigating high-temperature structure of HPTD. At present, the origin of the noncentrosymmetricity in HPTD nematic glass is an open question and we are aware that other explanations of the observed phenomenon could also be proposed.

\section{CONCLUSIONS}

The temperature-dependent studies of the nonlinear optical properties of HPTD - the nonchiral nematogen placed in an electric optical micro cell-demonstrated an unexpected nonvanishing second-order optical susceptibility below $90{ }^{\circ} \mathrm{C}$. Since SHG was not observed in the nematic phase of the compound even in the presence of static electric field, the appearance of the NLO properties in the temperature region of $90-60{ }^{\circ} \mathrm{C}$ is attributed to spontaneous and reproducible formation of polar noncentrosymmetric nanocrystallites within the nematic glassy and polycrystalline phases. The intensity of the observed SHG signal increases 
as the viscosity of the glass increases, reaching a maximum at about $50{ }^{\circ} \mathrm{C}$ below the $\mathrm{Cr}-\mathrm{N}$ transition. Molecular ordering in the glassy phase of HPTD leading to the appearance of noncentrosymmetricity is an interesting phenomenon, whose nature is still unresolved. Even though the net spontaneous polar order appears to be only a small fraction of the maximum available effect, the spontaneous emergence of noncentrosymmetricity may be of practical significance when compared to other ways of inducing a polar order in assemblies of nonlinear molecules, for example, electric field poling or all-optical poling, which also lead to only fractional alignment of the NLO chromophores and have many deficiencies in practical applications. Further research is necessary to determine whether the observed effect is related to the growth of polar nanocrystallites within the glassy nematic phase and dependent on the role of equilibrium between the cis and trans forms of HPTD during temperature cycling.

\section{ACKNOWLEDGMENTS}

The authors acknowledge the Australian Research Council Discovery Project No. DP0556942 and the NSF Grant No. DMR-0111657 for financial support. They thank Dr. Adam Januszko for his help in the preparation of the cells for optical measurements, and Professor Barry Luther-Davies and Dr. Vesselin Kolev of Laser Physics Centre at ANU for their help with the laser system.

${ }^{1}$ Optical Applications of Liquid Crystals, edited by L. Vicari (Institute of Physics, Philadelphia, PA, 2003).

${ }^{2}$ The Optics of Thermotropic Liquid Crystals, edited by S. Elston and R. Sambles (Taylor \& Francis, London, 1998).

${ }^{3}$ Liquid Crystals: Applications and Uses, edited by B. Bahadur (World Scientific, Singapore, 1990), Vols. 1-3.

${ }^{4}$ I.-C. Khoo, Liquid Crystals: Physical Properties and Nonlinear Optical Phenomena (Wiley, New York, 1995).
${ }^{5}$ L. S. Goldberg and J. M. Schnur, Appl. Phys. Lett. 14, 306 (1969).

${ }^{6}$ R. Macdonald, F. Kentischer, P. Warnick, and G. Heppke, Phys. Rev. Lett. 81, 4408 (1998).

${ }^{7}$ Molecular Nonlinear Optics: Materials, Physics, and Devices, edited by J. Zyss (Academic, San Diego, CA, 1994).

${ }^{8}$ D. S. Hermann et al., Liq. Cryst. 24, 295 (1998).

${ }^{9}$ S. Sprunt, J. Naciri, B. R. Ratna, R. Shashidar, B. K. Bihari, J. Kumar, and S. K. Tripathy, Appl. Phys. Lett. 66, 1443 (1995).

${ }^{10}$ B. Park, M. Lim, J. H. Lee, J. H. Kim, and S. D. Lee, Ferroelectrics 179 , 231 (1996)

${ }^{11}$ J. Etxebarria, C. L. Folcia, N. Pereda, and J. Ortega, Phys. Rev. E 57, 5634 (1998).

${ }^{12}$ N. Pereda, C. L. Folcia, J. Etxebarria, and J. Ortega, Liq. Cryst. 26, 375 (1999).

${ }^{13}$ P. Kaszynski and A. G. Douglass, J. Organomet. Chem. 581, 28 (1999).

${ }^{14} \mathrm{P}$. Kaszynski, in Anisotropic Organic Materials: Approaches to Polar Order, ACS Symposium Series Vol. 798, edited by R. Glaser and P. Kaszynski (American Chemical Society, Washington, DC, 2001), p. 68.

${ }^{15} \mathrm{P}$. Kaszynski and D. Lipiak, in Materials for Optical Limiting, edited by R. Crane, K. Lewis, E. Van Stryland, and M. Khoshnevisan (MRS, Boston, MA, 1995), Vol. 374 p. 341; P. Kaszynski, D. Lipiak, K. A. Bairamov, E. Brady, M. K. Patel, and J. Laska, in Advances in Boron Chemistry (Royal Society of Chemistry, Cornwall, UK, 1997), p. 507.

${ }^{16}$ R. Swanepoel, J. Opt. Soc. Am. A 2, 1339 (1985).

${ }^{17}$ V. Chandrasekharan and H. Damany, Appl. Opt. 7, 687 (1968).

${ }^{18}$ J. C. Manifacier, J. Gasiot, and J. P. Fillard, J. Phys. E 9, 1002 (1976).

${ }^{19}$ T. Ohtsuka, H. Ohnishi, and H. Takatsu, in Physical Properties of Liquid Crystals: Nematics, edited by D. A. Dunmur, A. Fukuda, and G. R. Luckhurst (IEE, London, 2001), p. 515, and references therein.

${ }^{20}$ A. Samoc, M. Samoc, B. Luther-Davies, V. Z. Kolev, R. K. Bagien, X. Luo, and C. Zha, Mol. Cryst. Liq. Cryst. 446, 123 (2006).

${ }^{21}$ A. Samoc et al., Proc. SPIE 4798, 87 (2002).

${ }^{22}$ A. Samoc, A. Holland, M. Tsuchimori, O. Watanabe, M. Samoc, B. Luther-Davies, and V. Z. Kolev, Proc. SPIE 5949, 59490X1 (2005).

${ }^{23}$ M. J. S. Dewar and M. L. McKee, Inorg. Chem. 19, 2662 (1980).

${ }^{24}$ M. B. Feller, W. Chen, and Y. R. Shen, Phys. Rev. A 43, 6778 (1991).

${ }^{25}$ J. Jerphagnon and S. K. Kurtz, J. Appl. Phys. 41, 1667 (1970).

${ }^{26}$ R. L. Sutherland, D. G. McLean, and K. Sean, Handbook of Nonlinear Optics, 2nd ed. (Dekker, New York, 2003).

${ }^{27}$ K. D. Singer, M. G. Kuzyk, and J. E. Sohn, J. Opt. Soc. Am. B 4, 968 (1987). 